\title{
Franco Montagna
}

\author{
AN ALGEBRAIC TREATMENT \\ OF IMPRECISE PROBABILITIES
}

\begin{abstract}
This is a survey paper about an algebraic approach to imprecise probabilities. In the first part of it, we outline the work by Walley on imprecise probabilities and the more algebraic approach of Fedel et al.. Then, in the second part we will present some work in progress about a general treatment of upper and lower probabilities over many-valued events and of upper and lower previsions of gambles, by means of Universal Algebra.
\end{abstract}

\section{Interpretation of probability in terms of bets}

There are several approaches to probability. A first approach is based on the notion of frequence. According to this approach, the probability of an event is its relative frequence on a large number of experiments. In our opinion, although very useful, frequence cannot serve as a definition of probability: first of all, in general it is impossible to reproduce several times an experiment in exactly the same circumstances, and second, in general it is not possible to reproduce the same experiment a large number of times. For instance, in order to compute the probability that a bridge lasts for, say, 200 years, one should build a huge number of bridges, all in the same place, and then wait for 200 years.

Bruno de Finetti $[\mathrm{dF}]$ proposed a radically different interpretation of probability in terms of bets: the probability of an event $\phi$ is the betting odd $\alpha \in[0,1]$ that a rational player $\mathrm{P}$ would accept for the following game:

GAME (1). Player $\mathrm{P}$ chooses his betting odd $\alpha$ for $\phi$. The opponent $\mathrm{O}$ chooses a (possibly negative) amount of money $\lambda$.

Then, $\mathrm{O}$ pays $\lambda \alpha$ to $\mathrm{P}$ and gets back $\lambda v(\phi)$, where $v(\phi)$ is the truth value of $\phi$.

We stipulate that paying $\lambda<0$ means receiving $-\lambda$ and receiving $\lambda<0$ means paying $-\lambda$.

2000 Mathematics Subject Classification: 06D35, 60A86. 
The coherence criterion is given not for a single event but for a set of events.

Let $S$ be a finite set of events, and let for each $\phi \in S, \alpha_{\phi}$ be a betting odd for $\phi$ (intuitively, $\alpha_{\phi}$ represents the subjective probability assigned by $\mathrm{P}$ to the event $\phi)$. The function assigning to each $\phi \in S$ the number $\alpha_{\phi}$ is said to be an assessment.

A system of bets is defined to be a map $\phi \mapsto \lambda_{\phi}$ from $S$ into $\mathbf{R}$ (intuitively, $\lambda_{\phi}$ represents the amount of money that $\mathrm{O}$ is willing to bet on $\phi$ ).

Then, de Finetti's interpretation can be extended to the following game:

GAME (2). Let $\phi \mapsto \alpha_{\phi}$ be the assessment chosen by player P. Then, the opponent $\mathrm{O}$ chooses his system of bets $\phi \mapsto \lambda_{\phi}$ on $S$. The payoff of $\mathrm{P}$ will be $\sum_{\phi \in S} \lambda_{\phi}\left(\alpha_{\phi}-v(\phi)\right)$, where as usual $v(\phi)$ represents the truth value of $\phi$.

An assessment is said to be coherent if there is no system of bets $\phi \mapsto \lambda_{\phi}$ which (may be individually acceptable, but) taken altogether causes $\mathrm{P}$ a sure loss, that is, such that $\sum_{\phi \in S} \lambda_{\phi}\left(\alpha_{\phi}-v(\phi)\right)<0$ for every valuation $v$.

REMARK. It is readily seen that the non-existence of a system of bets leading $\mathrm{P}$ to a sure loss also implies the non-existence of a system of bets leading $\mathrm{O}$ to a sure loss. I.e., coherence prevents both players from the possibility of a sure loss.

It turns out that this coherence criterion leads to the properties of a probability distribution.

Theorem 1. (de Finetti) An assessment $\phi \mapsto \alpha_{\phi}: \phi \in S$, where $S$ is a finite set of events is coherent iff there is a probability distribution on the algebra of events generated by $S$ which extends it.

EXAMPLE. Consider the assessment $\Lambda: p \mapsto \frac{1}{2}, \neg p \mapsto \frac{1}{3}$. Clearly, $\Lambda$ cannot be extended to a probability distribution. Now suppose that $\mathrm{O}$ bets one euro both on $p$ and on $\neg p$. In this case, $\mathrm{P}$ gets $\frac{1}{2}+\frac{1}{3}=\frac{5}{6}$ euro and, since either $p$ or $\neg p$ will be true, he has to pay 1 euro. It follows that this system of bets causes to $\mathrm{P}$ a sure loss.

\section{Probabilities over many-valued events}

In [Mu1], the author extends de Finetti's theorem to many-valued events. Although de Finetti believed that an event must be either completely true or completely false, there are several reasons to consider many-valued events and their probabilities.

First of all, many-valued events (like e.g., the market will be stable next week, or there will be much traffic on the highway) occur very often in the real life and influence our decisions. Such events force us to use intermediate 
values. For instance, it is almost impossible to find a threshold for the minimum number of cars necessary to make the event there will be much traffic in the highway true.

Second, even though intermediate values are sometimes associated to vagueness, in many situations they allow for a more precise description of the event. Suppose e.g. that we are drawing a die and that we want to bet about the event the outcome will be high (high for a die, of course). Then, we may agree that e.g., 4, 5 and 6 are high and 1, 2 and 3 are not, but using intermediate values we may obtain a better description of the event: for instance, we may assign to the sentence the outcome is high the truth value $\frac{n-1}{5}$ where $n$ is the outcome, and hence we can say not only that 1 is not high and 6 is high, but also that 2 is high with degree $\frac{1}{5}, 3$ is high with degree $\frac{2}{5}$, etc.

Third, many-valued events may be interpreted as special cases of random variables (namely, as special random variables taking values in $[0,1]$ ). We will see that many-valued events are not so special, in the sense that we may somehow treat any bounded random variable by means of many-valued events. We will return to this point in the later sections of this paper.

In the case of many-valued events, the situation is quite similar to that of Game (2), (and the payoff formula is formally the same), the only difference being that now the truth value $v(\phi)$ of an event is not necessarily 0 or 1 , but it may be an intermediate value.

Also the coherence criterion remains unchanged (no sure loss for P).

To close the circle, we need an analogue of a probability distribution for many-valued events. In this new situation, events are no longer boolean, and hence we have to choose a logic in which events can be treated.

There may be several many-valued logics for expressing many-valued events and their probability, but our preferred logic is Łukasiewicz logic, the main reason being that this is the only fuzzy logic whose connectives are all continuous.

Modulo this choice, the most natural extension of the concept of probability measure is the concept of state over an MV-algebra.

Although we do not give an explicit axiomatization here, we recall that the class of $M V$-algebras is the variety (in the sense of universal algebra) generated by the algebra $([0,1], \oplus, \neg, 0,1)$ where $x \oplus y=\min \{x+y, 1\}$ and $\neg x=1-x$. This algebra will be denoted by $[0,1]_{M V}$.

Other important MV-operations are $x \odot y=\neg(\neg x \oplus \neg y), x \ominus y=x \odot \neg y$, $x \wedge y=x \odot(\neg x \oplus y)$ and $x \vee y=x \oplus(y \odot \neg x)$.

In $[0,1]_{M V}, x \odot y=\max \{x+y-1,0\}, x \ominus y=\max \{x-y, 0\}, x \wedge y=$ $\min \{x, y\}$ and $x \vee y=\max \{x, y\}$. 
MV-algebras constitute the equivalent algebraic semantics of Łukasiewicz logic in the sense of Blok and Pigozzi, cf. [Ha]. In other words, MV-algebras stand to Eukasiewicz logic as Boolean algebras stand to classical logic.

A state on an MV-algebra $\mathbf{A}$ (cf. [Mus]) is a function $s$ from $\mathbf{A}$ into $[0,1]$ such that $s(1)=1$ and whenever $x \odot y=0$, then $s(x \oplus y)=s(x)+s(y)$.

An important theorem, proved independently by Panti and Kroupa says that states may be represented as integrals. More precisely, let $\mathbf{A}$ be an MValgebra, and let $X_{A}$ be the set of all homomorphisms from $\mathbf{A}$ into $[0,1]_{M V}$ (also called valuations) with the topology of pointwise convergence (i.e., a set $Y$ of valuations is closed iff the pointwise limit of any pointwise converging generalized sequence of valuations in $Y$ is in $Y$ ). Then $X_{A}$ becomes a compact Hausdorff space.

To any element $a$ of $\mathbf{A}$ we associate the function $a^{*}$ from $X_{A}$ into $[0,1]$, defined, for all $v \in X_{A}$ by $a^{*}(v)=v(a)$. It can be proved that every $a^{*}$ is continuous. Note that the map $a \mapsto a^{*}$ is one-one iff $\mathbf{A}$ is semisimple (in the sense of Universal Algebra).

The next theorem, proved independently by Panti and by Kroupa, says the following:

Theorem 2. $[\mathrm{Pa}],[\mathrm{Kr}]$ For every state $s$ on $\mathbf{A}$ there is a unique Borel regular probability measure $\mu$ on $X_{A}$ such that for all $a \in \mathbf{A}, s(a)=\int_{X} a^{*} d \mu$.

Hence, states can be regarded as very natural generalizations of probability measures.

Now Mundici [Mu1] generalizes de Finetti's theorem as follows:

THEOREM 3. An assessment on an arbitrary set $S$ of many-valued events (regarded as elements of an $M V$-algebra $\mathbf{A}$ ) is coherent iff there is a state on A which extends it.

\section{Random variables and expected values}

Following de Finetti, Walley [Wa] considers probability over events as a special case of expected values (previsions) of bounded random variables (gambles). Clearly, gambles can take any real value, possibly negative. Thus, the betting odds may be negative.

Game (2) can be extended to this new situation in the obvious way. (Now events are replaced by gambles and can take any real value. Hence, valuations are now maps from random variables into the reals). Once again, coherence is expressed in terms of no sure loss.

What is the appropriate mathematical apparatus for representing random variables (gambles)?

In Walley's approach, the basic structure is assumed to be a vector space over the the reals, possibly with a Hausdorff topology. 
In our more algebraic approach (cf. [FKMR]), gambles are elements of a divisible lattice ordered abelian group with strong unit. ${ }^{1}$

We recall that a lattice ordered abelian group (in the sequel, $\ell$-group) is an abelian group equipped with lattice operations $\vee$ and $\wedge$ which distribute over the group operation $+: x+(y \vee z)=(x+y) \vee(x+z)$ and similarly for $\wedge$, cf. $[\mathrm{BKW}]$.

A strong unit of an $\ell$-group is an element $u>0$ such that for every element $x$ of the $\ell$-group there is a natural number $n$ such that $x \leq n u$ ( $n u=u+\ldots+u, n$ times).

An $\ell$-group is said to be divisible if for every positive integer $n$ and for every $x$ there is a (necessarily unique) $y$, usually denoted by $\frac{x}{n}$, such that $x=n y$. Divisible $\ell$ groups are vector spaces over the rational field.

Expected values on a divisible $\ell$-group $\left(\mathbf{G}, 1_{G}\right)$ with strong unit $1_{G}$ are precisely the positive normalized linear functionals $E$ from $\mathbf{G}$ into $\mathbf{R}$ (this means $E(x) \geq 0$ when $x \geq 0, E\left(1_{G}\right)=1$ and $E(\lambda x+\mu y)=\lambda E(x)+\mu E(x)$ for all rational $\lambda$ and $\mu$ ).

By a famous theorem of Mundici, $[\mathrm{Mu} 0]$, given an $\ell$-group $\left(\mathbf{G}, 1_{G}\right)$ with strong unit $1_{G}$, its $\left[0_{G}, 1_{G}\right]$ interval carries an MV-algebra, denoted by $\Gamma\left(\mathbf{G}, 1_{G}\right)$, with $x \oplus y=(x+y) \wedge 1_{G}$ and $\neg x=1_{G}-x$. Moreover, all $\mathrm{MV}$-algebras arise in this way.

More precisely, letting, for every homomorphism $h$ of $\ell$-groups with strong unit from $\left(\mathbf{G}, 1_{G}\right)$ into $\left(\mathbf{H}, 1_{H}\right), \Gamma(h)$ be the restriction of $h$ to $\Gamma\left(\mathbf{G}, 1_{G}\right)$, we have that $\Gamma$ is a functor from the category of $\ell$-groups with strong unit into the category of MV-algebras (with morphisms the homomorphisms). Finally, $\Gamma$ has a two-sided adjoint $\Gamma^{-1}$, such that the pair $\left(\Gamma, \Gamma^{-1}\right)$ is an equivalence of categories.

MV-algebras of the form $\Gamma\left(\mathbf{G}, 1_{G}\right)$, where $\mathbf{G}$ is a divisible $\ell$ group with strong unit $1_{G}$ are called divisible. In a divisible MV-algebra one has operations $\frac{x}{n}$ such that $n \frac{x}{n}=x$ and $\frac{x}{n} \odot(n-1) \frac{x}{n}=0$.

For divisible MV-algebras the connection with $\ell$-groups with strong unit is even stronger: indeed, for every element $g \in \mathbf{G}$, there are an element $a \in \Gamma\left(\mathbf{G}, 1_{G}\right)$, an integer $z$ and a natural number $n$ such that $g=n a+z$ (where if $z= \pm k, k$ a natural number, $z$ stands for $\pm k 1_{G}$ ). Moreover, operations on $\mathbf{G}$ can be described by means of the operation of $\Gamma\left(\mathbf{G}, 1_{G}\right)$. For instance, $(n a+z)+(m b+u)=M\left(\frac{a}{M} \oplus \frac{b}{M}\right)+(z+u)$, where $M=n+m+1$.

Previsions over a divisible $\ell$-group with strong unit $\left(\mathbf{G}, 1_{G}\right)$ are connected with states on the underlying $\mathrm{MV}$-algebra $\Gamma\left(\mathbf{G}, 1_{G}\right)$ : the restriction of a prevision to $\left[0_{G}, 1_{G}\right]$ is a state on $\Gamma\left(\mathbf{G}, 1_{G}\right)$, and given a state $s$, the functional

\footnotetext{
${ }^{1}$ More precisely, in [FKMR], previsions and imprecise previsions are investigated, and results are obtained, under the weaker assumption of 2-divisibility, which roughly means that every element can be divided by 2 .
} 
$E$ defined by $E(n x+z)=n s(x)+z\left(\right.$ where $x \in \Gamma\left(\mathbf{G}, 1_{G}\right), n$ is a natural number and $z$ is an integer $)$ is a prevision on $\Gamma\left(\mathbf{G}, 1_{G}\right)$. Mundici's theorem naturally translates to:

Theorem 4. (cf. [Wa], but in a different framework) An assessment $\phi \mapsto \alpha_{\phi}$ over random variables from $S \subseteq \mathbf{G}$ (where $\mathbf{G}$ is a divisible $\ell$-group with strong unit $1_{G}$ ) is coherent iff it can be extended to a prevision on $\left(\mathbf{G}, 1_{G}\right)$.

\section{Upper and lower probabilities, upper and lower previsions}

There are many reasons for introducing imprecise probabilities or imprecise previsions, and there are many ways to do that, cf. [Hal], [Wa] and [AL]. Usually, imprecision is related to lack of knowledge, as shown in the following examples.

EXAMPLE. A box contains 100 marbles. 30 of them are red, and the remaining 70 are either red or blue (no more information is known). While it seems reasonable to say that the probability of choosing a red marble is $30 \%$, it is not so clear what the probability of choosing a blue marble is. For instance, the remaining marbles might be all blue, and then the probability would be $70 \%$, or even all yellow, and then the probability would be 0 . One might choose the mean value $35 \%$, but this last assessment is not as convincing as assigning probability $30 \%$ to the event: the chosen marble will be red.

EXAMPLE. I have to choose a betting odd for an event $\phi$, and I have no information about its probability. Hence, I consult three experts. Their assessments are $60 \%, 50 \%$ and $40 \%$ respectively. Accordingly, it seems to be safe to accept bets on $\phi$ as a bookmaker if the betting odd is $60 \%$ or more and to accept to bet on $\phi$ as a bettor if the betting odd is $40 \%$ or less. For intermediate betting odds, it may be safer not to bet at all.

This second example suggests the following interpretation of lower and upper probabilities or previsions. That is, upper probabilities (upper previsions) should be the suprema of sets of states (previsions), and lower probabilities (lower previsions) should be infima of sets of states (previsions).

Let us make the mathematical situation clearer (for concepts of Functional Analysis, cf. [Ru]).

(1) Let $X_{G}$ be the set of all homomorphisms from $\left(\mathbf{G}, 1_{G}\right)$ into $(\mathbf{R}, 1)$, and let for $g \in G, g^{*}: X_{G} \mapsto \mathbf{R}$ be defined by $g^{*}(v)=v(g)$. We will assume $\mathbf{G}$ to be semisimple, so that the map $g \mapsto g^{*}$ is one-one. Then random variables become continuous real-valued functions on the compact Hausdorff space $X_{G}$ (with the topology of pointwise convergence). 
Random variables form a topological vector lattice over $\mathbf{Q}$, that is, a vector space equipped with lattice operations and with a topology for which the operations of the algebra are continuous (with respect to the topology induced by the sup norm).

By the Stone-Waierstrass theorem the domain of such a topological vector space is uniformly dense in $C\left(X_{G}, \mathbf{R}\right)$ (the space of continuous functions from $X_{G}$ into $\mathbf{R}$ with the topology of uniform convergence).

(2) It follows that previsions have a unique extension to a normalized positive linear functional on $C\left(X_{G}, \mathbf{R}\right)$. Hence, they may be considered as elements of $C\left(X_{G}, \mathbf{R}\right)^{*}$ (the dual space of $C\left(X_{G}, \mathbf{R}\right)$ ), equipped with the weak * topology, that is, the smallest topology such that for all $f \in C\left(X_{G}, \mathbf{R}\right)$, the evaluation map $f^{*}(F)=F(f)\left(F \in C\left(X_{G}, \mathbf{R}\right)^{*}\right)$, is continuous.

(3) Every convex and closed set $\Sigma$ of previsions determines an upper prevision $u=u_{\Sigma}$ defined by $u(f)=\sup \{E(f): E \in \Sigma\}$ and a lower prevision $l=l_{\Sigma}$ defined by $l(f)=\inf \{E(f): E \in \Sigma\}=-u(-f)$. Note that since $\Sigma$ is closed, suprema and infima are actually attained and hence they are maxima and minima respectively.

Upper and lower previsions defined in this way, as suprema and infima of closed and convex sets of previsions, can be axiomatized as follows, [FKMR] (cf. also [Wa] for another equivalent axiomatization):

(1) $x \leq y$ implies $u(x) \leq u(y)$ (monotonicity).

(2) $u(f+q)=u(f)+q, q$ a rational constant (strong normalization).

(3) $u(q f)=q u(f)$, for every positive rational number $q$ (homogeneity).

(4) $u(f+g) \leq u(f)+u(g)$ (sublinearity).

(5) The lower prevision $l$ associated to $U$ is defined by $l(f)=-u(-f)$.

Previsions are precisely the upper previsions $u$ which coincide with their associated lower prevision, i.e., $u(x)=-u(-x)=l(x)$.

THEOREM 5. ([FKMR], [Wa]) Upper and lower previsions are in bijection with closed convex sets of previsions. In other words:

Upper previsions $u$ and their associated lower previsions $l(x)=-u(-x)$ are precisely the maps of the form $u(x)=\max \{E(x): E \in \Sigma\}$ and $l(x)=$ $\min \{E(x): E \in \Sigma\}$ for some closed convex set $\Sigma$ of previsions.

\section{Upper previsions, lower previsions and bets}

We consider the following interpretation of upper and lower previsions in terms of bets.

(GAME 3). Player $P$ selects an assessment $\phi \mapsto \alpha_{\phi}$ on a finite set $S$ of random variables. The number $\alpha_{\phi}$ represents the upper prevision of $\phi$ 
according to $\mathrm{P}$. Then his opponent $\mathrm{O}$ may bet only non-negative numbers $\lambda_{\phi}$ on each $\phi \in S$. The payoff for $\mathrm{P}$ will be $\sum_{\phi \in S} \lambda_{i}\left(\alpha_{\phi}-v(\phi)\right)$.

The absence of a system of bets causing to $\mathrm{P}$ a sure loss is a necessary condition for coherence, but not a sufficient condition. To the contrary, the coherence criterion for Game (3) is the non-existence of a bad bet for $\mathrm{O}$, that is a bet $\lambda_{\phi} \geq 0$ on $\phi \in S$ such that there is a system of bets $\lambda_{\phi_{i}} \geq 0$ on $\phi_{i} \in S, i=1, \ldots, n$ which gives $\mathrm{O}$ a better payoff, that is, such that $\sum_{i=1}^{n} \lambda_{\phi_{i}}\left(v\left(\phi_{i}\right)-\alpha_{\phi_{i}}\right)>\lambda_{\phi}\left(v(\phi)-\alpha_{\phi}\right)$ for every valuation $v$.

Note that the existence of a strategy causing $\mathrm{P}$ a sure loss implies the existence of a bad bet (indeed, in that case, betting nothing would be a bad bet). But it is easy to prove that there are assessments which do not cause a sure loss but admit bad bets, as shown in the next example.

EXAMPLE. Let $p$ and $q$ be propositional variables, and consider the assessment $p \mapsto \frac{1}{3}, q \mapsto \frac{1}{3}, p \oplus q \mapsto 1$. Then, there is no system of (non-negative) bets causing to $\mathrm{P}$ a sure loss. Indeed, if both $p$ and $q$ take value 0 , then $\mathrm{P}$ cannot lose money. However, betting any positive amount of money $\lambda$ on $p \oplus q$ is a bad bet, because betting the same amount of money on $p$ and on $q$ separately guarantees to $\mathrm{P}$ a strictly better payoff, independently of the valuation.

The existence of a bad for $\mathrm{O}$ does not mean that $\mathrm{O}$ is not rational (indeed, $\mathrm{O}$ need not choose that bad bet). It rather means that $\mathrm{P}$ was not rational, because he could make his assessment more attractive for $\mathrm{O}$ by reducing the betting odd $\alpha_{\phi}$ without any loss of money when $\mathrm{O}$ plays a rational strategy. For instance, in the example above, $\mathrm{P}$ might change his betting odd on $p \oplus q$ from 1 to $\frac{2}{3}$ without loss of money when $\mathrm{O}$ avoids bad bets.

To the contrary, the non existence of a bad bet implies that $\mathrm{P}$ cannot reduce his betting odds without a potential loss of money, even if $\mathrm{O}$ plays a rational strategy.

We point out that for a bookmaker it is important to choose a book which is not only relatively safe, but also attractive for the bettors (otherwise, nobody would bet on it).

A similar approach was proposed by Walley, who considers the point of view of the bettor rather than the point of view of the bookmaker. Hence, his coherence criterion becomes (essentially) the non-existence of a good bet, that is, a bet for which there is an alternative system of bets causing to the bettor a worse payoff independently of the valuation.

In any case:

THEOREM 6. ([FKMR]) Let $\Pi: \phi \mapsto \alpha_{\phi}$ be an assessment on a finite set $S$ of elements of a divisible $\ell$-group with strong unit. Then: 
(1) $\Pi$ can be extended to an upper prevision iff there is no bad bet for $O$ in Game (3).

(2) $\Pi$ can be extended to a lower prevision if there is no good bet for $O$ in Game (3).

(3) $\Pi$ can be extended to an expected value function (i.e., to a prevision) if there is neither a good bet nor a bad bet for $O$ in Game (3).

\section{Upper and lower probabilities}

Upper probabilities $u$ and lower probabilities $l$ are just restrictions of upper and lower previsions to $[0,1]$-valued gambles. They can be axiomatized in the language of divisible MV-algebras as follows:

(1) For all $x, u(x) \in[0,1]$ and $u(1)=1$ (normalization).

(2) $u(x \oplus y) \leq u(x)+u(y)$ when $x \odot y=0$ (sublinearity).

(3) $u(q x)=q u(x)$ for each rational $q$ in $[0,1]$ (homogeneity).

(4) $x \leq y$ implies $u(x) \leq u(y)$ (monotonicity).

(5) $u(x \oplus q)=u(x)+q$ for each rational $q$ in $[0,1]$ such that $x \odot q=0$ (strong normalization).

(6) Finally, the associated lower probability $l$ is defined by $l(x)=1-u(\neg x)$.

In this way, states are precisely those upper probabilities $u$ which coincide with their associated lower probabilities $l(x)=1-u(\neg x)$.

Of course, the interpretation in terms of bets of upper and lower previsions extends to upper and lower probabilities over MV-algebras as well, and we have an analogue of Theorem 6 for upper and lower probabilities, cf. [FKMR].

We also stress that upper (lower) probabilities are closely related to upper (lower) previsions:

(1) Given an upper (lower) prevision $U$ on a divisible $\ell$-group with strong unit $\left(\mathbf{G}, 1_{G}\right)$, its restriction $u$ to $\Gamma\left(\mathbf{G}, 1_{G}\right)$ is an upper (lower) probability on $\Gamma\left(\mathbf{G}, 1_{G}\right)$.

(2) Conversely, given a divisible $\mathrm{MV}$-algebra $\mathbf{A}$ and an upper (lower) probability on it, every element of $\Gamma^{-1}(\mathbf{A})$ can be written as $n x+z$ for some integer $z$, for some natural number $n$ and for some $x \in \mathbf{A}$. Moreover, the operator $U$ on $\Gamma^{-1}(\mathbf{A})$ defined by $U(n x+z)=n u(x)+z$ is well defined and it turns out to be an upper (lower) prevision on $\Gamma^{-1}(\mathbf{A})$.

\section{A logic for reasoning on imprecise probabilities and on upper and lower previsions}

Based on the fact that coherence has something to do with logic (a coherent assessment is an assessment which does not suffer an evident lack of 
rationality, and hence coherence is a sort of absence of contradiction), we want to find a logic for reasoning about upper and lower probabilities, in which we can express coherence in terms of logical coherence. We will work inside an equational logic (or, semantically speaking, in a variety). The appropriate variety for the treatment of upper and lower probabilities is the variety of $U M V$-algebras, which are divisible MV-algebras with an operation $u$ such that:

(1) $u(1)=1$.

(2) $u(q x)=q u(x)$ for every rational number $q$ in $[0,1]$.

(3) $u(x \oplus y) \leq u(x) \oplus u(y)$.

(4) $u(x \wedge y) \leq u(x)$.

(5) $u(q \oplus y)=q \oplus u(y \ominus(q \odot y))$.

(6) $u(t)=t$ whenever $t$ is a term whose variables occur only under the scope of $u .^{2}$

Of course, in any UMV-algebra one also has a lower probability operator $l$, defined by $l(x)=\neg u(\neg x)$.

The difference with upper and lower probabilities on an MV-algebra is that now the external operators $u$ and $l$ from $\mathbf{A}$ into $[0,1]$ are replaced by internal operations on A. Hence, UMV-algebras are algebras in the sense of Universal Algebra. Moreover, their axioms are all equational, and hence UMV-algebras constitute a variety of universal algebras.

We briefly comment about the axioms of UMV-algebras. Axioms (1) ... (4) reflect normality, homogeneity, sublinearity and monotonicity of $u$, respectively.

Axiom (5) reflects the fact that if $q$ is a rational and $q \odot y=0$, then $u(q \oplus y)=q \oplus u(y)$.

As regards to Axiom (6), if all variables occur in $t$ under the scope of $u$, then $t$ represents an event with constant truth value, which must coincide with its upper probability.

That UMV-algebras fit our purposes is witnessed by the following result:

THEOREM 7. There is a computable function associating to every rationalvalued assessment $\Pi: \phi_{i} \mapsto \alpha_{i}: i=1, \ldots, n$ over a UMV-algebra three finite sets of equations $\Pi_{U}, \Pi_{L}$ and $\Pi_{S}$ such that:

(1) $\Pi_{U}$ is satisfiable (in the variety of UMV-algebras) iff there is no bad bet based on $\Pi$.

(2) $\Pi_{L}$ is satisfiable iff there is no good bet based on $\Pi$.

(3) $\Pi_{S}$ is satisfiable iff there is neither a good bet nor a bad bet based on $\Pi$.

\footnotetext{
${ }^{2}$ As noted by Yde Venema, Axiom (6) may be formulated as a finite schema, namely, $u(\neg u(t))=\neg u(t)$, and $u\left(u\left(t_{1}\right) \oplus u\left(t_{2}\right)\right)=u\left(t_{1}\right) \oplus u\left(t_{2}\right)$.
} 


\section{A logical treatment of gambles and of upper and lower previsions}

One may wonder if there is a similar logic for treating gambles and upper and lower previsions. The appropriate class seems to be the class of $U G$ algebras, namely, of divisible $\ell$-groups with strong unit equipped with an internal upper prevision satisfying suitable axioms. We can express all the basic properties of gambles and of their upper and lower previsions by means of equations, except from the existence of a strong unit, which is not even a first-order property. Hence, we will follow another approach, that is, we will interpret both gambles and upper (lower) previsions inside UMV.

We will start from the semantical level. Mundici's categorical equivalence between $\mathrm{MV}$-algebras and $\ell$-groups with strong unit extends to an equivalence between the category of UMV-algebras and the category of UGalgebras.

The proof of this equivalence is based on the fact that every internal upper probability on a divisible $\mathrm{MV}$-algebra has a unique extension to an upper prevision on its enveloping $\ell$-group, which makes it into a UG-algebra, and every homomorphism of UMV-algebras has a unique extension to a homomorphism of its corresponding UG-algebra.

This semantical equivalence can be turned into a syntactical equivalence, provided that the elements of a UG-algebras are presented in the form $n x+z$, $n$ a natural number, $z$ an integer and $x$ an element between 0 and the strong unit. In this way, terms of UG-algebras can be simulated by means of terms of UMV-algebras. In particular, the upper prevision $U$ is expressed in terms of the upper probability $u$ by the formula $U(n x+z)=n u(x)+z$. Finally, UGequations are translated into UMV-equations, and the translation preserves finite semantic consequence.

The opposite translation, from UG-algebras into UMV-algebras, is straightforward. Hence, we get:

THEOREM 8. There is a computable function associating to every rational valued assessment $\Pi: \phi_{i} \mapsto \alpha_{i}: i=1, \ldots, n$ over random variables, thought of as members of a UG-algebra, three finite sets of equations $\Pi_{U}, \Pi_{L}$ and $\Pi_{S}$ over UMV-algebras such that:

(1) $\Pi_{U}$ is satisfiable iff there is no bad bet based on $\Pi$.

(2) $\Pi_{L}$ is satisfiable iff there is no good bet based on $\Pi$.

(3) $\Pi_{S}$ is satisfiable iff there is neither a good bet nor a bad bet based on $\Pi$.

\section{Complexity issues}

An $S M V$-algebra (cf. [FM]) is a UMV-algebra satisfying $u(x)=\neg u(\neg x)$. In $[\mathrm{BF}]$, the authors prove: 
THEOREM 9. (1) The coherence problem for probabilistic assessments (i.e., absence of both a bad bet and a good bet) is NP-complete.

(2) The satisfiablity problem for SMV-equations without nested occurrences of $u$ is NP-complete.

It is not hard to generalize the above theorem to the case of MV-algebras A equipped with several independent operators $u_{1}, \ldots, u_{n}$ (instead of just one) such that for $i=1, \ldots, n,\left(\mathbf{A}, u_{i}\right)$ is an SMV-algebra. Let us denote by $\mathrm{SMV}_{n}$ the resulting equational logic. Then it is possible to interpret UMVequations with $n$ occurrences of $u$ at most and without nested occurrences of $u$ into $S M V_{n}$ (the idea is that in $\operatorname{SMV}_{n}$ we can define $u(x)=u_{1}(x) \vee \ldots \vee$ $\left.u_{n}(x)\right)$. It follows:

THEOREM 10. (1) The coherence problem for assessments of imprecise probabilities (i.e., absence of a bad bet) is NP-complete. The same holds for the absence of good bets.

(2) The satisfiability problem for UMV-equations without nested occurrences of $u$ is NP-complete.

\section{References}

[AL] B. Anger, J. Lembcke, Infinite subadditive capacities as upper envelopes of measures, Zeitschrift für Wahrscheinlichkeitstheorie 68 (1985), 403-414.

[BKW] A. Bigard, K. Keimel, S. Wolfenstein, Groupes et Anneaux Réticulés, Lecture Notes in Math. 608 (1977), Springer Verlag.

[BF] S. Bova, F. Flaminio, The coherence of Eukasiewicz assessments is NP-complete, Internat. J. Approx. Reason. 51(3) (2010), 294-304.

[dF] B. de Finetti, Theory of Probability, vol. I. John Wiley and Sons, Chichester, 1974.

[FKMR] M. Fedel, K. Keimel, F. Montagna, W. Roth, Imprecise probabilities, bets and functional analytic methods in Eukasiewicz logic, submitted.

[FM] T. Flaminio, F. Montagna, $M V$-algebras with internal states and probabilistic fuzzy logics, Internat. J. Approx. Reason. 50 (2009), 138-152.

[Ha] P. Hájek, Metamathematics of Fuzzy Logic, Kluwer, Dordrecht, 1998.

[Hal] J. Y. Halpern, Reasoning about Uncertainty, MIT Press, 2003.

[Kr] T. Kroupa, Every state on a semisimple MV algebra is integral, Fuzzy Sets and Systems 157(20) (2006), 2771-2782.

[Mu0] D. Mundici, Interpretation of $A F C^{*}$-algebras in Eukasiewicz sentential calculus, J. Funct. Anal. 65 (1986), 15-63.

[Mus] D. Mundici, Averaging the truth value in Eukasiewicz logic, Studia Logica 55(1) (1995), 113-127.

[Mu1] D. Mundici, Bookmaking over infinite-valued events, Internat. J. Approx. Reason. 46 (2006), 223-240.

[Pa] G. Panti, Invariant measures in free $M V$ algebras, Comm. Algebra 36 (2008), 2849-2861. Available at Arxiv preprint math. LO/0508445, 2005.

[Ru] W. Rudin, Functional Analysis, 2nd Edition, McGraw-Hill Publ., 1991. 
[Wa] P. Walley, Statistical Reasoning with Imprecise Probabilities, Volume 42 of Monographs on Statistics and Applied Probability, Chapman and Hall, London 1991.

DEPARMENT OF MATHEMATICS AND COMPUTER SCIENCE "R. MAGARI" UNIVERSITY OF SIENA

Pian dei Mantellini 44

53100 SIENA, ITALY

E-mail: montagna@unisi.it

Received September 9, 2010. 